\title{
V-parameter Study of Silica-Air 1D Photonic Crystal Fiber by Modulating Geometrical Parameters at Different Optical Communication Ranges
}

\author{
Arka Karmakar, Indrajit Roy and Arpan Deyasi
}

\begin{abstract}
Electromagnetic wave propagation inside one-dimensional photonic crystal fiber is numerically characterized by computing V-parameter, cladding index and effective mode profiles. Dependence on these parameters on geometrical structure of the fiber reflects the design tolerance, and corresponding modal characteristics. Air-hole spacing and periodicity are varied to observe the effect on V-parameter; along with effective core radius, which is a function of periodicity, is modified to see the effect on cladding index and effective mode profiles. Results obtained from empirical relations method are compared with analytical formula of Nielsen and Mortensen. Periodicity of the structure is regarded as numerically equal to the central wavelength of the desired range throughout the computation. Refractive index of the core is taken as independent of operating wavelength. Simulated parameters have critical importance to estimate the characteristics of photonic crystal fiber which will help the design engineers to study the performance at $870 \mathrm{~nm}, 1330 \mathrm{~nm}$ and $1550 \mathrm{~nm}$.
\end{abstract}

Keywords--- V-Parameter Cladding Index, Effective Core Radius, Single Mode Propagation, Empirical Relations Method, Optical Communication

\section{INTRODUCTION}

$\mathrm{P}$ HOTONIC crystal is a periodic arrangement of multilayer structure where propagation of electromagnetic wave is governed by change of refractive indices of those dielectric materials. Photonic crystal fibers are developed on the physics

Arka Karmakar, Undergraduate Student, Departmen of Electronics \& Communication Engineering, West Bengal University of Technology, Kolkata, India, karmakararka@gmail.com

Indrajit Roy, Undergraduate Student, Departmen of Electronics \& Communication Engineering, West Bengal University of Technology, Kolkata, India, indrajitroy23@gmail.com

Arpan Deyasi, Assistant Professor, Departmen of Electronics \& Communication Engineering, West Bengal University of Technology, Kolkata, India,deyasi_arpan@yahoo.co.in

DOI: 10.9756/BIJRCE.3111 required for the primary purpose of optical communication. This enables to achieve the distant dreams, may not be possible with conventional fiber technology. These are also called microstructured fibers; consist of solid core with airholes arranged in general triangular lattice format along the axis of the core. Effective modulation of geometrical parameters of PCF's such as air-hole spacing, pitch, air-hole diameter etc, will provide a greater flexibility from the application point of view in the domain of optical communication [1], sensing [2], high power technology [3], optical nonlinearity [4], integrated photonics [5], quantum information science [6]. It may be considered as a revolution in the next generation communication scenario.

Effective cladding index is defined as the largest modal index of the photonic crystal structure without any defect states; has a critical importance in determining the optical performance [7]. It is the effective index of the fundamental space filling mode, and is a function of the operating wavelength. It influences the single-mode behavior [8], bending loss [9], dispersion properties [10] of the optical fiber. Silica as a material for deign of photonic crystal fiber was proposed much earlier [11], and it is also demonstrated that suitable deposition of different materials changes the modal properties [12]; which is important for biosensor applications. Importance of single mode propagation is emphasized by Birks [13], and for that purpose, V-parameters plays an important role. Later Cregan analyzed the same when propagation is considered through air [14]. Numerical methods are already applied by eminent researchers [15] to study waveguiding properties for higher order modes. Effect of effective core area was also studied on field distributions at optical communication frequency ranges [16]. Experimental investigation was also carried out [17] to observe the effect of geometrical parameters on optical properties at desired frequency range.

Nielsen [18] first provided some analytical formulae for computation of $\mathrm{V}$ parameters of photonic crystal fiber, and Baggett [19] made a comparative study with conventional optical fiber. It is already established by researchers that PCF can provide far better performance precisely form the point of view of dispersion and loss than conventional fiber. Different designs are also prescribed by researchers [20] to improve the performance. In general, triangular core pattern is considered for simulation and experimental works, and present work is 
also based on that structure. Empirical relations method use the fitting coefficients obtained form experimental results, and thus it can safely be considered as the first-hand performance predicting method for PCF.

In the present paper, V-parameter of air-silica photonic crystal fiber is computed for single mode propagation as a function of operating wavelength for three different optical communication range, and for each case, periodicity of the micro structured fiber is taken numerically equal to the centre wavelength. Empirical relations method is considered for numerical analysis, and results are compared with analytical findings as predicted by Nielsen [18]. Air-hole spacing is considered inside solid core in a triangular manner, and effective cladding index and effective mode outlines are also calculated as function of operating wavelength. Refractive index of the core is taken as independent of operating wavelength. Simulated results provide the performance of the one-dimensional finite periodic crystal for optical applications.

\section{MATHEMATICAL MODELING}

We consider a photonic crystal fiber with triangular lattice pattern of holes as shown in Fig 1, where ' $d$ ' is the hole, $\Lambda$ is hole pitch or periodicity of the structure, $\mathrm{n}_{\text {core }}$ is the refractive index of core material.

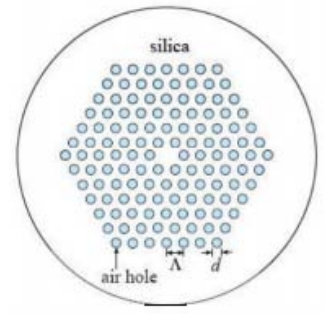

Figure 1: Schematic Diagram of Triangular Lattice Pattern in PCF

For simplicity of mathematical computation, we neglect the effect of hole at the center. In order to investigate the single-mode condition, fundamental mode is required to be solved over a wide range of wavelengths. In a conventional fiber, the number of bound modes is governed by V- number, which increases with decrease of wavelength. For such pattern, V-parameter can be given as-

$$
V=\frac{2 \pi}{\lambda} \alpha_{\text {eff }}\left(n_{\text {core }}^{2}-n_{f s m}^{2}\right)^{0.5}
$$

where we assume that $\alpha_{\text {eff }}$ i.e., the effective core radius is a function of periodicity, $\mathrm{n}_{\text {core }}$ is the core index, $\mathrm{n}_{\mathrm{fsm}}$ is cladding layer index .

For V-parameter, the $\mathrm{V}$ values can be calculated as a function of $\lambda / \Lambda$ and $d / \Lambda$. This can be represented as-

$$
V=A_{1}+\frac{A_{2}}{1+A_{3} \exp \left[\frac{A_{4} \lambda}{\Lambda}\right]}
$$

where fitting coefficients are given by-

$$
\begin{aligned}
& A_{i}=\alpha_{i 0}+\alpha_{i 1}\left(\frac{d}{\Lambda}\right)^{\kappa_{i 1}}+\alpha_{i 2}\left(\frac{d}{\Lambda}\right)^{\kappa_{i 2}}+\alpha_{i 3}\left(\frac{d}{\Lambda}\right)^{\kappa_{i 3}} \\
& B_{i}=\beta_{i 0}+\beta_{i 1}\left(\frac{d}{\Lambda}\right)^{\delta_{i 1}}+\beta_{i 2}\left(\frac{d}{\Lambda}\right)^{\delta_{i 2}}+\beta_{i 3}\left(\frac{d}{\Lambda}\right)^{\delta_{i 3}}
\end{aligned}
$$

Knowledge on V parameters can be obtained from Eq. (2), where the coefficients can be derived from Eq. (3) and Eq. (4) respectively. The fitting coefficients are obtained from experimental results for the present material composition. Cladding indices can be determined from Eq. (1) when core refractive index is given for a specified PCF structure. For single mode propagation, boundary condition is taken as -

$V<2.405$

V-parameter was earlier computed using analytical method considering its dependence on wavelength, hole spacing and periodicity, given as

$$
V=\frac{A(d / \Lambda)}{1+\left[B(d / \Lambda) \exp \left(C(d / \Lambda) \frac{\lambda}{\Lambda}\right)\right]}
$$

where $\mathrm{A}, \mathrm{B}, \mathrm{C}$ are fitting coefficients.

\section{RESULTS \& DISCUSSIONS}

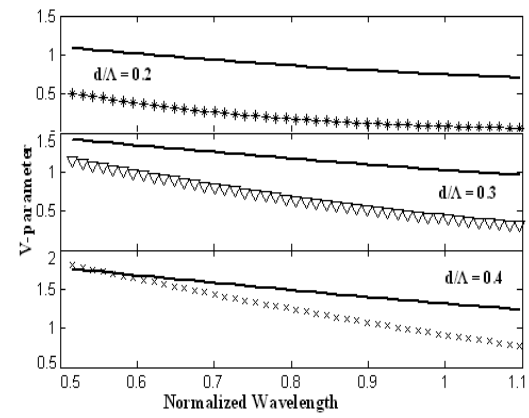

Figure 2: V-parameter Profile with Normalized Wavelength for Different Hole Dimension to Periodicity Ratio when Periodicity is considered as $1550 \mathrm{nmCalculation}$ of $\mathrm{V}$ parameter using empirical relations method for silica-air hole photonic crystal fiber is performed assuming single mode propagation, and wavelength range is chosen from $0.8 \mu \mathrm{m}$ to $1.7 \mu \mathrm{m}$ for optical communication applications. Results are compared with analytical findings.

Fig 2 shows the V-parameter profile with normalized wavelength when periodicity of the structure is considered as $1550 \mathrm{~nm}$. From the plot, it may be observed that with increasing dimension of the air-hole, there is better agreement between empirical relations method and Nielsen formula for 
single mode propagation. The deviation increases as we increase the operating wavelength.

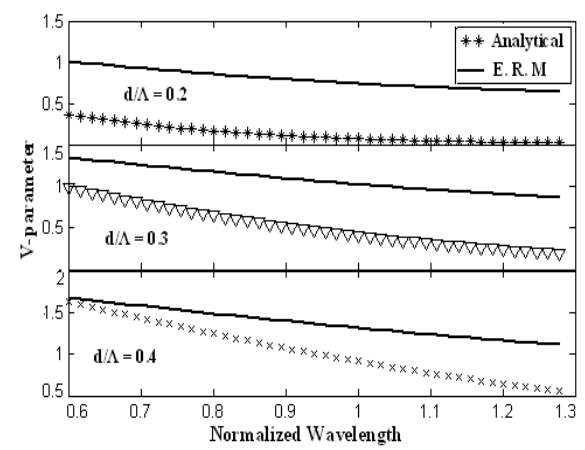

Figure 3: V-parameter Profile with Normalized Wavelength for Different Hole Dimension to Periodicity Ratio when Periodicity is considered as $1330 \mathrm{~nm}$

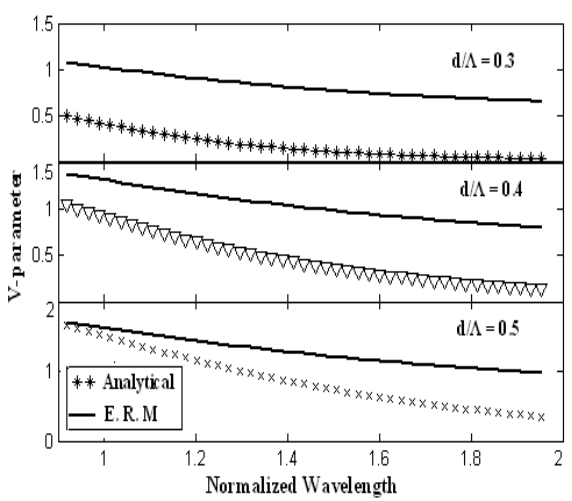

Figure 4: V-Parameter Profile with Normalized Wavelength for Different Hole Dimension to Periodicity Ratio when Periodicity is Considered as $870 \mathrm{~nm}$

Fig 3 \& Fig 4 give the V-parameter profile for $1330 \mathrm{~nm}$ and $870 \mathrm{~nm}$ periodicity respectively. A comparative study shows that for a particular ratio of hole dimension to periodicity, with decrease of operating wavelength, vparameter reduces. It becomes almost zero for $870 \mathrm{~nm}$ when $\mathrm{d} / \Lambda$ is 0.2 , and hence the result is omitted as it carries hardly any significance. Thus it may be concluded that Nielsen formula is applicable for a considerable dimension of hole, and also of lower wavelength, i.e., higher frequency region.

It is well-known that $1550 \mathrm{~nm}$ is the most suitable optical spectrum for communication purpose, and present day fibers are designed at that range of interest. Hence once $\mathrm{V}$ parameter is calculated, cladding index and effective mode profiles are estimated for the same hole diameter to hole pitch ratio, and comparative study are graphically displayed in Fig. 5 \& Fig 6. From Fig. 5, it can be concluded that with smaller hole diameter for a given periodic structure, refractive index of cladding material remains almost constant, whereas it drastically detoriates for higher value of hole diameter. Same is effective for effective mode profile, as evident form Fig 6.

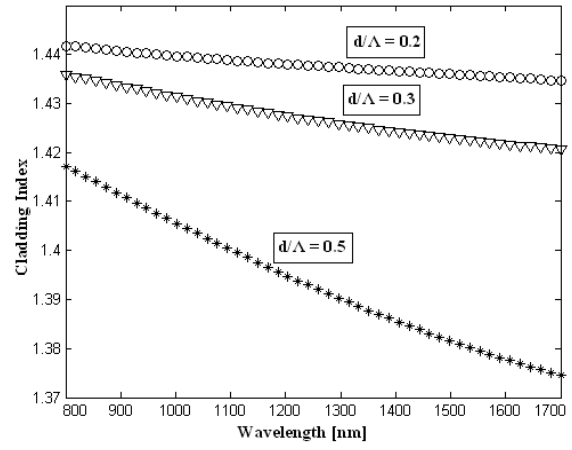

Figure 5: Cladding Index Profile with Wavelength for Different Hole Spacing when Periodicity of the Structure is $1550 \mathrm{~nm}$

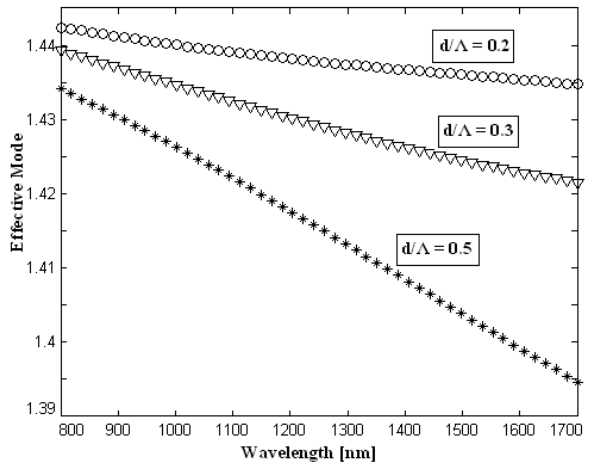

Figure 6: Effective Mode Profile with Wavelength for Different Hole Spacing when Periodicity of the Structure is $1550 \mathrm{~nm}$

For different effective core radius, cladding index and effective mode profiles are computed as shown in Fig 7 \& Fig 8. Periodicity of the structure is made equal to $1550 \mathrm{~nm}$, and effective core radius is assumed as function of periodicity only. It can be observed from Fig. 7 that for lower wavelength region, cladding index rapidly decreases, whereas for larger wavelength region, it begins to saturate. Completely different nature is observed from effective mode profile, as shown in Fig. 8. Rate of reduction of effective mode is faster for higher wavelength region, and it is more pronounced for lower effective core radius. It may be observed that at required wavelength, cladding index starts to saturate irrespective of the magnitude of effective core radius, but it depends heavily on it as far the effective mode is concerned. 


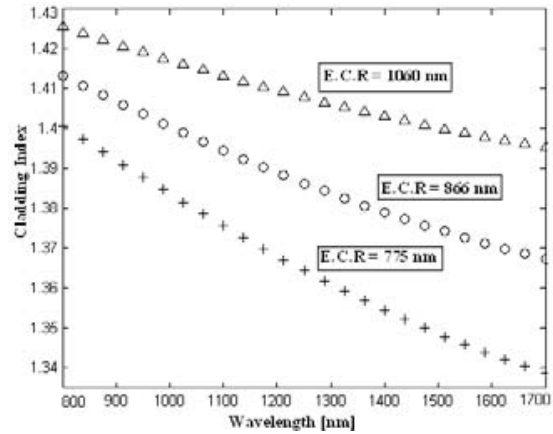

Figure 7: Cladding Index Profile with Wavelength for Different Effective Core Radius when Periodicity of the Structure is $1550 \mathrm{~nm}$

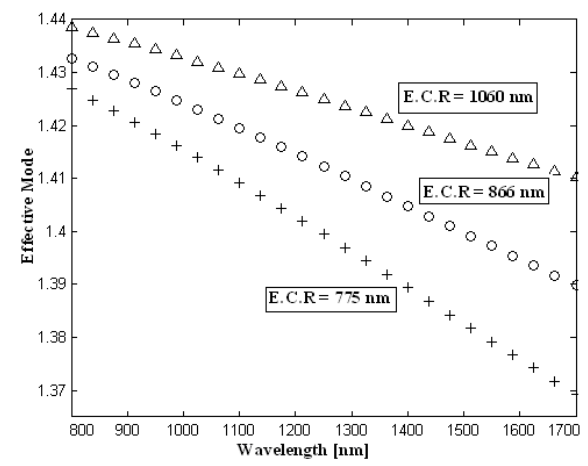

Figure 8: Effective Mode Profile with Wavelength for Different Effective Core Radius when Periodicity of the Structure is $1550 \mathrm{~nm}$

\section{CONCLUSION}

The subscript for V-parameter of one-dimensional photonic crystal fiber is computed using empirical relations method and results are compared with analytical outcome, as prescribed by Nielsen. Results are calculated for three different optical wavelengths i.e., $870 \mathrm{~nm}, 1330 \mathrm{~nm}$ and 1550 $\mathrm{nm}$. Effective mode profile and cladding index profile of a 1D photonic crystal fiber is numerically computed from the estimation of V-parameter for different hole spacing when periodicity of the structure is considered as $1550 \mathrm{~nm}$. Also the parameters are calculated for different effective core radius. Air-hole spacing is varied to observe the effect on those parameters. Effective core radius is considered as a function of periodicity, which is taken as numerically equal to the central wavelength. Refractive index of the core is taken as independent of operating wavelength. The simulated profiles will help the design engineers to fabricate one-dimensional photonic crystal fiber with $\mathrm{SiO}_{2}$-air composition at various wavelengths as required for optical communication purpose with greater accuracy.

\section{REFERENCES}

[1] J. Hansryd, P. A. Andrekson, M. Westlund, J. Li and P. O. Hedekvist, "Fiber-based Optical Parametric Amplifiers and their Applications," IEEE Journal of Selected Topics on Quantum Electronics., Vol. 8, Pp. 506-520, 2002.
[2] W. Belhadj, F. AbdelMalek and H. Bouchriha, "Characterization and Study of Photonic Crystal Fibres with Bends," Material Science and Engineering: C, Vol. 26, Pp. 578-579, 2006.

[3] J. Limpert, T. Schreiber, S. Nolte, H. Zellmer, T. Tunnermann, R. Iliew, F. Lederer, J. Broeng, G.Vienne, A. Petersson and C. Jakobsen, "High Power Air-Clad Large-Mode-Area Photonic Crystal Fiber Laser," Optic Express, Vol. 11, Pp. 818-823, 2003.

[4] J. Limpert, A. Liem, M. Reich, T. Schreiber, S. Nolte, H. Zellmer, A. Tünnermann, J. Broeng, A. Petersson and C. Jakobsen, "LowNonlinearity Single-Transverse-Mode Ytterbium-Doped Photonic Crystal Fiber Amplifier," Optic Express, Vol. 12, Pp. 1313-1319, 2004.

[5] K. Bayat, G. Z. Rafi, G. S. A. Shaker, N. Ranjkesh, S. K. Chaudhuri and S. Safavi-Naeini, "Photonic-Crystal based Polarization Converter for Terahertz Integrated Circuit", IEEE Transactions on Microwave Theory and Techniques, Vol. 58, Pp. 1976-1984, 2010.

[6] H. Azuma, "Quantum Computation with Kerr-Nonlinear Photonic Crystals", Journal of Physics D: Applied Physics, Vol. 41, p. 025102, 2008.

[7] D. Mogilevstev, T. A. Birks and P. St. J. Russell, "Localized Function Method for Modeling Defect Modes in 2-D Photonic Crystals", Journal of Lightwave Technology, Vol. 17, Pp. 2078-2081, 1999.

[8] K. Saitoh and M. Koshiba, "Single-Polarization Single-Mode Photonic Crystal Fibers," IEEE Photonics Technology Letters, Vol. 15, Pp. 13841386, 2003.

[9] M. D. Nielsen, N. A. Mortensen, M. Albertsen, J. R. Folkenberg, A. Bjarklev and D. Bonacinni, "Predicting Macrobending Loss for LargeMode Area Photonic Crystal Fibers," Optic express, Vol. 12, Pp. 17751779, 2004.

[10] R. K. Sinha and S. K. Varshney, "Dispersion Properties of Photonic Crystal Fibers", Microwave and Optical Technology Letters, Vol. 37, Pp. 129-132, 2003.

[11] J. C. Knight, T. A. Birks, P. S. J. Russell and D. M. Atkin, "All-Silica Single-Mode Optical Fiber with Photonic Crystal Cladding," Optical Letters, Vol. 21, Pp. 1547-1549, 1996.

[12] M. Smietana, D. Brabant, W. J. Bock, P. Mikulic, T. Eftimov, "Inline Core-Cladding Intermodal Interferometer based on Nano-Coated Photonic Crystal Fiber for Refractive-Index Sensing", Proc. SPIE, 77531R, 2011.

[13] T. A. Birks, J. C. Knight and P. St. J. Russell, "Endlessly Single-Mode Photonic Crystal Fiber," Optical Letters, vol. 22, pp. 961-963, 1997.

[14] R. F. Cregan, B. J. Mangan, J. C. Knight, T. A. Birks, P. S. J. Russell, P. J. Roberts and D. C. Allan, "Single-Mode Photonic Band Gap Guidance of Light in Air," Science, vol. 285, pp. 1537-1539, 1999.

[15] Y. A. Mazhirina and L. A. Melnikov, "Numerical Modeling of Waveguiding Properties of Solid Core Photonic Crystal Fibers", Third International Workshop on Theoretical and Computational NanoPhotonics, Pp. 136-138, 2010.

[16] A. Rostami, A. Ghanbari, H. Soofi and F. Janabi-Sharifi, "Enlarging Effective Mode Area of Photonic Crystal Fibers using Defected Core Structures", International Symposium on Optomechatronic Technologies (ISOT), 2010.

[17] J. C. Knight, T. A. Birks, P. St. J. Russell and J. P. de Sandro, "Properties of Photonic Crystal Fiber and the Effective Index Model", Journal of the Optical Society of America A, Vol. 15, Pp. 748-752, 1998.

[18] M. Nielsen and N. Mortensen, "Photonic Crystal Fiber Design based on the V-parameter", Optic Express, Vol. 11, Pp. 2762-2768, 2003.

[19] J. C. Baggett, T. M. Monro, K. Furusawa and D. J. Richardson, "Comparative Study of Large-Mode Holey and Conventional Fibers", Optical Letters, Vol. 26, Pp. 1045- 2001.

[20] J. K. Ranka, R. S. Windeler, and A. J. Stentz, "Optical Properties of High-Delta Air-Silica Microstructure Optical Fibers", Optical Letters, Vol. 25, Pp. 796, 2000. 